\title{
Invariant Principal Order Ideals under Foata's Transformation
}

\author{
Teresa X.S. Li* \\ School of Mathematics and Statistics \\ Southwest University \\ Chongqing 400715, P.R. China \\ pmgb@swu.edu.cn
}

\author{
Melissa Y.F. Miao ${ }^{\dagger}$ \\ Center for Combinatorics, LPMC-TJKLC \\ Nankai University \\ Tianjin 300071, P.R. China \\ miaoyinfeng@mail.nankai.edu.cn
}

Submitted: Sep 1, 2012; Accepted: Oct 9, 2012; Published: Oct 18, 2012

Mathematics Subject Classifications: 05A05, 05A15, 05A19

\begin{abstract}
Let $\Phi$ denote Foata's second fundamental transformation on permutations. For a permutation $\sigma$ in the symmetric group $S_{n}$, let $\widetilde{\Lambda}_{\sigma}=\left\{\pi \in S_{n}: \pi \leqslant w \sigma\right\}$ be the principal order ideal generated by $\sigma$ in the weak order $\leqslant_{w}$. Björner and Wachs have shown that $\widetilde{\Lambda}_{\sigma}$ is invariant under $\Phi$ if and only if $\sigma$ is a 132-avoiding permutation. In this paper, we consider the invariance property of $\Phi$ on the principal order ideals $\Lambda_{\sigma}=\left\{\pi \in S_{n}: \pi \leqslant \sigma\right\}$ with respect to the Bruhat order $\leqslant$. We obtain a characterization of permutations $\sigma$ such that $\Lambda_{\sigma}$ are invariant under $\Phi$. We also consider the invariant principal order ideals with respect to the Bruhat order under Han's bijection $H$. We find that $\Lambda_{\sigma}$ is invariant under the bijection $H$ if and only if it is invariant under the transformation $\Phi$.
\end{abstract}

Keywords: Foata's second fundamental transformation; Han's bijection; Bruhat order; principal order ideal

\section{Introduction}

Let $S_{n}$ denote the symmetric group on $[n]=\{1,2, \ldots, n\}$. Foata's second fundamental transformation $\Phi$ on permutations in $S_{n}$ maps the major index of a permutation $\pi$ to the inversion number of $\Phi(\pi)$, see Foata [5]. Björner and Wachs [2] have shown that Foata's second fundamental transformation can also be used in the study of subsets $U$ of $S_{n}$ over which the inversion number and the major index are equidistributed. In particular, they

\footnotetext{
*Supported by Southwest University of China (Grant No. SWU112040).

${ }^{\dagger}$ Supported by the 973 Project, the PCSIRT Project of the Ministry of Education, and the National Science Foundation of China.
} 
showed that if the subset $U$ is an order ideal of permutations in $S_{n}$ with respect to the weak order, then $U$ is invariant under $\Phi$ if and only if the maximal elements of $U$ are 132-avoiding permutations.

In this paper, we investigate principal order ideals $\Lambda_{\sigma}=\left\{\pi \in S_{n}: \pi \leqslant \sigma\right\}$ with respect to the Bruhat order $\leqslant$ that are invariant under Foata's transformation. We obtain a characterization of permutations $\sigma$ for which $\Lambda_{\sigma}$ is invariant under $\Phi$.

We also consider the principal order ideals $\Lambda_{\sigma}$ that are invariant under Han's bijection [6] while restricted to permutations. Recall that Han's bijection, denoted $H$, is a Foatastyle bijection defined on words, which can be used to show that the Z-statistic introduced by Zeilberger and Bressoud [7] is Mahonian. We shall show that a principal order ideal $\Lambda_{\sigma}$ with respect to the Bruhat order is invariant under $H$ if and only if it is invariant under $\Phi$.

Let us give a brief review of Foata's transformation $\Phi$ and Han's bijection $H$ on permutations. To describe $\Phi$, we need to define a factorization for permutations on a finite set of positive integers. Let $A$ be a set of $n$ positive integers, and let $x$ be an integer not belonging to $A$. For any permutation $w=w_{1} w_{2} \cdots w_{n}$ on $A, x$ induces a factorization

$$
w=\gamma_{1} \gamma_{2} \cdots \gamma_{j}
$$

where each subword $\gamma_{i}(1 \leqslant i \leqslant j)$ is determined uniquely as follows:

(i) If $w_{n}<x$, then the last element of $\gamma_{i}$ is smaller than $x$ and all the remaining elements of $\gamma_{i}$ are greater than $x$;

(ii) If $w_{n}>x$, then the last element of $\gamma_{i}$ is greater than $x$ and all the remaining elements of $\gamma_{i}$ are smaller than $x$.

For example, let $w=387125$. Then the factorization induced by $x=4$ is $w=38 \cdot 7 \cdot 125$, while the factorization induced by $x=6$ is $w=3 \cdot 871 \cdot 2 \cdot 5$, where we use dots to separate the factors.

For a factorization $w=\gamma_{1} \gamma_{2} \cdots \gamma_{j}$ induced by $x$, let

$$
\delta_{x}(w)=\gamma_{1}^{\prime} \gamma_{2}^{\prime} \cdots \gamma_{j}^{\prime}
$$

where $\gamma_{i}^{\prime}(1 \leqslant i \leqslant j)$ is obtained from $\gamma_{i}$ by moving the last element to the beginning of $\gamma_{i}$. For example, for the permutation $w=387125$, based on the above factorization, we have $\delta_{4}(w)=837512$.

To define $\Phi$, we still need the $k$-th $(1 \leqslant k \leqslant n)$ Foata bijection $\phi_{k}: S_{n} \longrightarrow S_{n}$. Let $\sigma=\sigma_{1} \sigma_{2} \cdots \sigma_{n} \in S_{n}$. For $k=1$, define $\phi_{k}(\sigma)=\sigma$. For $k>1$, define

$$
\phi_{k}(\sigma)=\delta_{\sigma_{k}}\left(\sigma_{1} \sigma_{2} \cdots \sigma_{k-1}\right) \cdot \sigma_{k} \sigma_{k+1} \cdots \sigma_{n} .
$$

The transformation $\Phi$ is defined to be the composition $\phi_{n} \circ \phi_{n-1} \circ \cdots \circ \phi_{1}$, that is, for $\sigma \in S_{n}$

$$
\Phi(\sigma)=\phi_{n}\left(\cdots \phi_{2}\left(\left(\phi_{1}(\sigma)\right)\right) \cdots\right)
$$


We next describe Han's bijection $H$ on permutations, and we need two maps $C_{x}$ and $C^{x}$ $(x \in[n])$ from the set of permutations on $[n] \backslash\{x\}$ to $S_{n-1}$. Assume that $w=w_{1} w_{2} \cdots w_{n-1}$ is a permutation on $[n] \backslash\{x\}$. Let $\tau_{i}=w_{i}-x(\bmod n)$, namely,

$$
\tau_{i}= \begin{cases}w_{i}-x+n, & \text { if } w_{i}<x \\ w_{i}-x, & \text { if } w_{i}>x\end{cases}
$$

and let

$$
\nu_{i}= \begin{cases}w_{i}, & \text { if } w_{i}<x \\ w_{i}-1, & \text { if } w_{i}>x\end{cases}
$$

The maps $C_{x}$ and $C^{x}$ are defined by

$$
C^{x}(w)=\tau_{1} \tau_{2} \cdots \tau_{n-1} \quad \text { and } \quad C_{x}(w)=\nu_{1} \nu_{2} \cdots \nu_{n-1} .
$$

It is easy to check that both $C^{x}$ and $C_{x}$ are one-to-one correspondences between the set of permutations on $[n] \backslash\{x\}$ and $S_{n-1}$. Let $\sigma=\sigma_{1} \sigma_{2} \cdots \sigma_{n} \in S_{n}$. The bijection $H$ can be defined recursively as follows

$$
H(\sigma)=C_{\sigma_{n}}^{-1}\left(H\left(C^{\sigma_{n}}\left(\sigma^{\prime}\right)\right)\right) \sigma_{n},
$$

where we set $H(1)=1$ and $\sigma^{\prime}=\sigma_{1} \sigma_{2} \cdots \sigma_{n-1}$. Note that the bijection $H$ can also be described in terms of permutation codes, see Chen, Fan and Li [3].

We now recall the Bruhat order on permutations. To describe this partial order, we need to clarify the definition of the multiplication of permutations. First, we regard a permutation $\pi \in S_{n}$ as a bijection on $[n]$ by setting $\pi(i)=\pi_{i}$. The product $\pi \sigma$ of two permutations $\pi, \sigma \in S_{n}$ is defined as the composition of $\pi$ and $\sigma$ as functions, that is, $\pi \sigma(i)=\pi(\sigma(i))$ for $i \in[n]$. For $1 \leqslant i<j \leqslant n$, let $(i, j)$ denote the transposition of $S_{n}$ that interchanges the elements $i$ and $j$. Thus, the multiplication on the right of a permutation $\pi$ by a transposition $(i, j)$ has the same effect as interchanging the elements $\pi_{i}$ and $\pi_{j}$. Similarly, the multiplication on the left of a permutation $\pi$ by a transposition $(i, j)$ is equivalent to the exchange of the elements $i$ and $j$. For example, for $\pi=236514$, we have $\pi(2,5)=216534$ and $(2,5) \pi=536214$.

The Bruhat order of $S_{n}$ is defined as follows. For two permutations $\pi, \sigma \in S_{n}$, we say that $\pi \leqslant \sigma$ if there exists a sequence of transpositions $\left(i_{1}, j_{1}\right),\left(i_{2}, j_{2}\right), \ldots,\left(i_{k}, j_{k}\right)$ such that

$$
\sigma=\pi\left(i_{1}, j_{1}\right)\left(i_{2}, j_{2}\right) \cdots\left(i_{k}, j_{k}\right)
$$

and

$$
\operatorname{inv}\left(\pi\left(i_{1}, j_{1}\right) \cdots\left(i_{t-1}, j_{t-1}\right)\right)<\operatorname{inv}\left(\pi\left(i_{1}, j_{1}\right) \cdots\left(i_{t}, j_{t}\right)\right), \text { for } t=1,2, \ldots, k,
$$

where $\operatorname{inv}(\pi)$ is the inversion number of $\pi$, namely,

$$
\operatorname{inv}(\pi)=\mid\left\{\left(\pi_{i}, \pi_{j}\right): 1 \leqslant i<j \leqslant n \text { and } \pi_{i}>\pi_{j}\right\} \mid .
$$


If replacing transpositions by adjacent transpositions in the above definition, then the Bruhat order reduces to the weak order $\leqslant_{w}$. Denote by $\Lambda_{\sigma}$ and $\widetilde{\Lambda}_{\sigma}$ the principal order ideals generated by $\sigma$ in the Bruhat order and the weak order respectively.

The following theorem gives a characterization of the covering relation in the Bruhat order.

Theorem 1 (Björner and Brenti [1], Lemma 2.1.4). Let $\pi, \sigma \in S_{n}$. Then $\pi$ is covered by $\sigma$ in the Bruhat order if and only if $\sigma=\pi(i, j)$ for some $1 \leqslant i<j \leqslant n$ such that $\pi_{i}<\pi_{j}$ and there does not exist $k$ such that $i<k<j$ and $\pi_{i}<\pi_{k}<\pi_{j}$.

The following theorem is due to Ehresmann [4], see also Björner and Brenti [1, Theorem 2.1.5], which gives a criterion for the comparison of two permutations in the Bruhat order. This characterization will be employed in the proof of Theorem 3. For a permutation $\pi \in$ $S_{n}$ and two integers $1 \leqslant i, j \leqslant n$, let $\pi[i, j]$ be the number of elements in $\left\{\pi_{1}, \pi_{2}, \ldots, \pi_{i}\right\}$ that are greater than or equal to $j$, that is,

$$
\pi[i, j]=\mid\left\{\pi_{k}: 1 \leqslant k \leqslant i \text { and } \pi_{k} \geqslant j\right\} \mid \text {. }
$$

Theorem 2 (Ehresmann [4]). Let $\pi, \sigma \in S_{n}$. Then $\pi \leqslant \sigma$ if and only if

$$
\pi[i, j] \leqslant \sigma[i, j]
$$

for any $1 \leqslant i, j \leqslant n$,

This paper is organized as follows. In Section 2, we present a characterization of permutations $\sigma$ such that the principal order ideals $\Lambda_{\sigma}$ are invariant under $\Phi$. Section 3 is devoted to the proof of the fact that $\Lambda_{\sigma}$ is invariant under Han's bijection $H$ if and only if it is invariant under Foata's transformation $\Phi$.

\section{Invariant principal order ideals under Foata's map}

The objective of this section is to give a characterization of invariant principal ideals $\Lambda_{\sigma}$ under Foata's transformation $\Phi$. To this end, we need a property on the maximal elements of the following subset $\Lambda_{\sigma}(k)$ of $\Lambda_{\sigma}$ which is defined by

$$
\Lambda_{\sigma}(k)=\left\{\tau: \tau \in \Lambda_{\sigma} \text { and } \tau_{n}=k\right\},
$$

where $1 \leqslant k \leqslant n$. It should be noted that by Theorem $2, \Lambda_{\sigma}(k)$ is nonempty unless $k \geqslant \sigma_{n}$. We need to consider a special element in $\Lambda_{\sigma}(k)$, denoted $M(\sigma, k)$. Define $M(\sigma, k)=\sigma$ if $k=\sigma_{n}$. The definition of $M(\sigma, k)$ for $k>\sigma_{n}$ can be described as follows.

Let $i_{1}$ be the largest element in $\sigma$ such that $i_{1}$ is to the right of $k$ and $\sigma_{n} \leqslant i_{1}<$ $k$. If $i_{1}=\sigma_{n}$, then define $M(\sigma, k)=\left(k, i_{1}\right) \sigma$. Otherwise, we continue to consider the permutation $\left(k, i_{1}\right) \sigma$. Let $i_{2}$ be the largest element in $\left(k, i_{1}\right) \sigma$ such that $i_{2}$ is to the right of $k$ and $\sigma_{n} \leqslant i_{2}<k$. If $i_{2}=\sigma_{n}$, then define $M(\sigma, k)=\left(k, i_{2}\right)\left(k, i_{1}\right) \sigma$. Otherwise, we consider the permutation $\left(k, i_{2}\right)\left(k, i_{1}\right) \sigma$, and let $i_{3}$ be the largest element in $\left(k, i_{2}\right)\left(k, i_{1}\right) \sigma$ 
such that $i_{3}$ is to the right of $k$ and $\sigma_{n} \leqslant i_{3}<k$. Repeating this procedure, we end up with an element $i_{s}(1 \leqslant s \leqslant n)$ such that $i_{s}=\sigma_{n}$. Define

$$
M(\sigma, k)=\left(k, i_{s}\right) \cdots\left(k, i_{2}\right)\left(k, i_{1}\right) \sigma .
$$

For example, for $\sigma=875169423, M(\sigma, 7)$ is constructed as follows. It is clear that $i_{1}=$ 6 . So, we have $\left(7, i_{1}\right) \sigma=865179423$. Now we see that $i_{2}=4$, and hence $\left(7, i_{2}\right)\left(7, i_{1}\right) \sigma=$ 865149723. Since $i_{3}=3=\sigma_{n}$, we find $M(\sigma, 7)=865149327$.

The following theorem will be employed in the proof of Theorem 5 .

Theorem 3. Suppose that $\sigma$ is a permutation in $S_{n}$ and $k$ is an integer such that $\sigma_{n} \leqslant$ $k \leqslant n$. Then, $M(\sigma, k)$ is the unique maximal element of $\Lambda_{\sigma}(k)$, that $i s, M(\sigma, k) \geqslant \tau$ for any $\tau \in \Lambda_{\sigma}(k)$.

Proof. It is clear that the theorem holds when $k=\sigma_{n}$. Now we consider the case $k>\sigma_{n}$. Assume that

$$
M(\sigma, k)=\left(k, i_{s}\right) \cdots\left(k, i_{2}\right)\left(k, i_{1}\right) \sigma .
$$

Let $\tau \in \Lambda_{\sigma}(k)$, and denote $\sigma^{(1)}=\left(k, i_{1}\right) \sigma$. To prove $\tau \leqslant M(\sigma, k)$, we first show that

$$
\tau \leqslant \sigma^{(1)}
$$

Let $m$ and $t$ be the indices such that $\sigma_{m}=k$ and $\sigma_{t}=i_{1}$. By the choice of $i_{1}$, we see that $1 \leqslant m<t \leqslant n$. It is easy to verify the following relation

$$
\sigma^{(1)}[i, j]= \begin{cases}\sigma[i, j]-1, & \text { if } m \leqslant i<t \text { and } i_{1}<j \leqslant k \\ \sigma[i, j], & \text { otherwise }\end{cases}
$$

Since $\tau[i, j] \leqslant \sigma[i, j]$ for $1 \leqslant i, j \leqslant n$, we see that (1) can be deduced from the following relation

$$
\tau[i, j]<\sigma[i, j], \quad \text { for } m \leqslant i<t \text { and } i_{1}<j \leqslant k .
$$

We now proceed to prove (3). We shall present detailed argument for the case $i=m$ and $j=i_{1}+1$ and the remaining cases can be dealt with in the same vein. Suppose to the contrary that $\tau\left[m, i_{1}+1\right]=\sigma\left[m, i_{1}+1\right]$. By the choice of $i_{1}$, we see that the elements $i_{1}+1, i_{1}+2, \ldots, k-1$ in $\sigma$ are all to the left of $\sigma_{m}$. Thus, we get

$$
\sigma\left[m, i_{1}+2\right]=\sigma\left[m, i_{1}+1\right]-1 .
$$

It follows that

$$
\tau\left[m, i_{1}+2\right] \geqslant \tau\left[m, i_{1}+1\right]-1=\sigma\left[m, i_{1}+1\right]-1=\sigma\left[m, i_{1}+2\right] .
$$

On the other hand, since $\tau \leqslant \sigma$, we see that $\tau\left[m, i_{1}+2\right] \leqslant \sigma\left[m, i_{1}+2\right]$. Hence,

$$
\tau\left[m, i_{1}+2\right]=\sigma\left[m, i_{1}+2\right]
$$


In a similar fashion, we find that

$$
\tau\left[m, i_{1}+3\right]=\sigma\left[m, i_{1}+3\right], \quad \tau\left[m, i_{1}+4\right]=\sigma\left[m, i_{1}+4\right], \quad \ldots \quad, \tau[m, k]=\sigma[m, k] .
$$

From the relation $\tau[m, k]=\sigma[m, k]$, we assert that the number $k$ belongs to the set $\left\{\tau_{1}, \tau_{2}, \ldots, \tau_{m}\right\}$. This can be seen as follows. Suppose to the contrary that $k \notin$ $\left\{\tau_{1}, \tau_{2}, \ldots, \tau_{m}\right\}$. Then, we have $\tau[m, k+1]=\tau[m, k]$. But, since $\sigma_{m}=k$, we get $\sigma[m, k+$ $1]=\sigma[m, k]-1$. Thus we obtain that $\tau[m, k+1]>\sigma[m, k+1]$, a contradiction.

Obviously, the above assertion that $k \in\left\{\tau_{1}, \tau_{2}, \ldots, \tau_{m}\right\}$ is contrary to the assumption that $\tau_{n}=k$. Thus, relation (3) holds for $i=m$ and $j=i_{1}+1$. This completes the proof of the relation in (1).

Using the same argument, we can deduce that

$$
\tau \leqslant\left(k, i_{2}\right) \sigma^{(1)}
$$

Repeating this procedure, we finally get

$$
\tau \leqslant\left(k, i_{s}\right) \cdots\left(k, i_{2}\right) \sigma^{(1)}=M(\sigma, k) .
$$

This completes the proof.

Before stating our main theorem, we need a result of Björner and Wachs [2] on the weak order. Let $\leqslant_{w}$ denote the weak order on permutations. For a given permutation $\sigma \in S_{n}$, they constructed a permutation $\gamma(\sigma) \in S_{n}$ such that $\gamma(\sigma) \leqslant w, \operatorname{inv}(\sigma) \leqslant \operatorname{maj}(\gamma(\sigma))$, and the last element of $\gamma(\sigma)$ is equal to $\sigma_{n}$. Moreover, they showed that $\operatorname{inv}(\sigma)=\operatorname{maj}(\gamma(\sigma))$ if and only if $\sigma$ is 132-avoiding. Recall that a permutation $\sigma$ is 132-avoiding if there do not exist numbers $1 \leqslant a<b<c \leqslant n$ such that $\sigma_{a}<\sigma_{c}<\sigma_{b}$. We shall not give the precise definition of $\gamma(\sigma)$, since we only require the properties of $\gamma(\sigma)$ as mentioned above.

The following lemma will be used in the proof of Theorem 5 .

Lemma 4. Suppose that $\sigma \in S_{n}$ is a permutation such that $\Lambda_{\sigma}$ is invariant under Foata's map $\Phi$. Then $\sigma$ is a 132-avoiding permutation.

Proof. Since $\Lambda_{\sigma}$ is invariant under $\Phi$, it is easy to see that $\Phi(\gamma(\sigma)) \leqslant \sigma$. Thus we have

$$
\operatorname{inv}(\Phi(\gamma(\sigma))) \leqslant \operatorname{inv}(\sigma)
$$

Recall that $\operatorname{inv}(\Phi(\gamma(\sigma)))=\operatorname{maj}(\gamma(\sigma))$. Hence, by (5), we deduce that maj $(\gamma(\sigma)) \leqslant$ $\operatorname{inv}(\sigma)$. On the other hand, as we have mentioned above, $\gamma(\sigma)$ possesses the property that $\operatorname{maj}(\gamma(\sigma)) \geqslant \operatorname{inv}(\sigma)$. So we get maj $(\gamma(\sigma))=\operatorname{inv}(\sigma)$. In other words, $\sigma$ is 132-avoiding. This completes the proof.

We are now ready to state the main result in this paper.

Theorem 5. Suppose that $\sigma$ is a permutation in $S_{n}$. Then the following assertions hold:

(1) If $\sigma_{n}=n$, then $\Lambda_{\sigma}$ is invariant under $\Phi$ if and only if $\Lambda_{\sigma^{\prime}}$ is invariant under $\Phi$, where

$$
\sigma^{\prime}=\sigma_{1} \sigma_{2} \cdots \sigma_{n-1}
$$


(2) If $\sigma_{n}=k<n$, then $\Lambda_{\sigma}$ is invariant under $\Phi$ if and only if

$$
\sigma=n(n-1) \cdots(k+1)(k-1) \cdots 21 k,
$$

where $\sigma=n(n-1) \cdots 21$ for $k=1$.

Proof. We first prove (1). Assume that $\Lambda_{\sigma}$ is invariant under Foata's map $\Phi$. To prove that $\Lambda_{\sigma^{\prime}}$ is invariant under $\Phi$, let $\pi^{\prime}$ be a permutation in $S_{n-1}$ such that $\pi^{\prime} \leqslant \sigma^{\prime}$. It is easy to see that $\pi^{\prime} n \leqslant \sigma^{\prime} n=\sigma$. Thus, $\Phi\left(\pi^{\prime} n\right) \leqslant \sigma$. Since $\Phi\left(\pi^{\prime} n\right)=\Phi\left(\pi^{\prime}\right) n$, we have

$$
\Phi\left(\pi^{\prime}\right) n \leqslant \sigma
$$

By the fact that $\sigma_{n}=n$, we find $\Phi\left(\pi^{\prime}\right) \leqslant \sigma^{\prime}$. Hence $\Lambda_{\sigma^{\prime}}$ is invariant under $\Phi$. Conversely, it can be easily checked that if $\Lambda_{\sigma^{\prime}}$ is invariant under $\Phi$, then $\Lambda_{\sigma}$ is invariant under $\Phi$.

Next, we proceed to prove (2). In this case, $\sigma_{n}=k<n$. Assume that $\sigma$ is a permutation in (6). It follows from Theorem 2 that

$$
\Lambda_{\sigma}=\left\{\pi \in S_{n}: \pi_{n} \geqslant k\right\}
$$

Let $\pi$ be a permutation in $\Lambda_{\sigma}$. Since the last element of $\Phi(\pi)$ is equal to $\pi_{n}$, from (7) we see that $\Phi(\pi)$ belongs to $\Lambda_{\sigma}$. So we deduce that $\Phi\left(\Lambda_{\sigma}\right) \subseteq \Lambda_{\sigma}$. Since $\Phi$ is a bijection, we have $\Phi\left(\Lambda_{\sigma}\right)=\Lambda_{\sigma}$, that is, $\Lambda_{\sigma}$ is invariant under $\Phi$.

It remains to prove the reverse direction of (2), that is, if $\Lambda_{\sigma}$ is invariant under $\Phi$, then $\sigma$ is a permutation of form (6). We have the following two cases.

Case 1: $\sigma_{n}=k=n-1$. We use induction on $n$. Assume that the assertion in (2) is true for permutations in $S_{n-1}$. Let $\sigma$ be a permutation in $S_{n}$ such that $\Lambda_{\sigma}$ is invariant under $\Phi$.

By Lemma 4, we see that $\sigma$ is 132 -avoiding. It is readily checked that $\sigma_{1}=n$. Let $\tau=(n-1, n) \sigma$. Evidently,

$$
\tau=M(\sigma, n) \quad \text { and } \quad \Lambda_{\tau}=\left\{\pi: \pi \leqslant \sigma, \pi_{n}=n\right\}
$$

which implies that $\Lambda_{\tau}$ is invariant under $\Phi$. Since $\tau_{n}=n$, by Part (1) of the theorem, we obtain that $\Lambda_{\tau^{\prime}}$ is invariant under $\Phi$, where $\tau^{\prime}=\tau_{1} \tau_{2} \cdots \tau_{n-1}$. Therefore, by the induction hypothesis, we deduce that

$$
\tau_{1} \tau_{2} \cdots \tau_{n-1}= \begin{cases}(n-1)(n-2) \cdots(i+1)(i-1) \cdots 21 i, & \text { if } \tau_{n-1}=i>1 \\ (n-1)(n-2) \cdots 21, & \text { if } \tau_{n-1}=1\end{cases}
$$

Now, we claim that $\tau_{n-1}=1$. Suppose to the contrary that $\tau_{n-1}=i>1$. Then we have

$$
\tau=(n-1)(n-2) \cdots(i+1)(i-1) \cdots 21 i n,
$$

and so

$$
\sigma=n(n-2) \cdots(i+1)(i-1) \cdots 21 i(n-1) .
$$


Consider the permutation

$$
\pi=(n-2) \cdots(i+1) i(i-1) \cdots 21 n(n-1) .
$$

Clearly,

$$
\begin{aligned}
\pi & \leqslant(n-2) \cdots(i+1) n(i-1) \cdots 21 i(n-1) \\
& \leqslant n(n-2) \cdots(i+1)(i-1) \cdots 21 i(n-1) \\
& =\sigma .
\end{aligned}
$$

However,

$$
\Phi(\pi)=n(n-2) \cdots(i+1) i(i-1) \cdots 21(n-1) \not<\sigma,
$$

which contradicts the assumption that $\Lambda_{\sigma}$ is invariant under $\Phi$. This completes the proof of the above claim that $\tau_{n-1}=1$.

We now arrive at the conclusion that $\tau=(n-1)(n-2) \cdots 21 n$. Hence

$$
\sigma=(n-1, n) \tau=n(n-2) \cdots 21(n-1),
$$

as required.

Case 2: $\sigma_{n}=k<n-1$. The proof is by induction on $\sigma_{n}$. Assume that the assertion is true for $\sigma_{n}>k$. We now consider the case $\sigma_{n}=k$.

To apply the induction hypothesis, we need to consider the principal order ideal generated by

$$
w=M(\sigma, k+1)=(k, k+1) \sigma .
$$

We first show that the principal order ideal $\Lambda_{w}$ has the following form

$$
\Lambda_{w}=\left\{\pi \in \Lambda_{\sigma}: \pi_{n} \geqslant k+1\right\}
$$

It is clear that $\Lambda_{w} \subseteq\left\{\pi \in \Lambda_{\sigma}: \pi_{n} \geqslant k+1\right\}$. It remains to show that

$$
\left\{\pi \in \Lambda_{\sigma}: \pi_{n} \geqslant k+1\right\} \subseteq \Lambda_{w}
$$

To prove (9), we need the permutation $\gamma(w)$. Keep in mind that the last element of $\gamma(w)$ is equal to $w_{n}$. This implies that $\gamma(w) \in \Lambda_{\sigma}(k+1)$. It follows that $\Phi(\gamma(w)) \in$ $\Lambda_{\sigma}(k+1)$. By Theorem 3, we see that $\Phi(\gamma(w)) \leqslant M(\sigma, k+1)=w$. Using the arguments in the proof of Lemma 4, we deduce that $w$ is 132-avoiding. Hence we conclude $\sigma^{-1}(i)<$ $\sigma^{-1}(k+1)$ for $i>k+1$.

In view of the construction of $M(\sigma, i)$, for any $i>k+1$, there exist integers $i_{1}>i_{2}>$ $\cdots>i_{s-2}>k+1$ such that

$$
\begin{aligned}
M(\sigma, i) & =(i, k)(i, k+1)\left(i, i_{s-2}\right) \cdots\left(i, i_{1}\right) \sigma \\
& \leqslant(i, k+1) M(\sigma, i) \\
& =(k, k+1)\left(i, i_{s-2}\right) \cdots\left(i, i_{1}\right) \sigma \\
& \leqslant(k, k+1) \sigma .
\end{aligned}
$$


Thus, for $i \geqslant k+1$ and $\pi \in \Lambda_{\sigma}(i)$, we have

$$
\pi \leqslant M(\sigma, i) \leqslant(k, k+1) \sigma=w,
$$

which implies the relation (9). Hence the proof of (8) is complete.

From (8), we see that $\Lambda_{w}$ is invariant under $\Phi$. By the induction hypothesis, we get

$$
w=n(n-1) \cdots(k+2) k(k-1) \cdots 21(k+1),
$$

which yields that

$$
\sigma=n(n-1) \cdots(k+2)(k+1)(k-1) \cdots 21 k .
$$

This completes the proof.

Theorem 5 has the following consequence.

Corollary 6. There are $\left(\begin{array}{l}n \\ 2\end{array}\right)+1$ permutations $\sigma$ in $S_{n}$ such that the principal order ideals $\Lambda_{\sigma}$ are invariant under Foata's transformation $\Phi$.

Proof. Let $a_{n}$ denote the number of principal order ideals in $S_{n}$ that are invariant under $\Phi$. By Theorem 5, it is easy to derive the following recurrence relation

$$
a_{n}=a_{n-1}+n-1
$$

Since $a_{1}=1$, the formula for $a_{n}$ is easily verified. This completes the proof.

For example, for $n=3$, there are four permutations $\sigma$ for which $\Lambda_{\sigma}$ is invariant under $\Phi: 123,213,312,321$. The following two figures are the Hasse diagrams of $\left(S_{3}, \leqslant\right)$ and $\left(S_{3}, \leqslant_{w}\right)$. The permutations $\sigma$ such that $\Lambda_{\sigma}$ is $\Phi$-invariant are written in boldface in Figure 1 , and the permutations $\sigma$ such that $\widetilde{\Lambda}_{\sigma}$ is $\Phi$-invariant are written in boldface as well in Figure 2 .

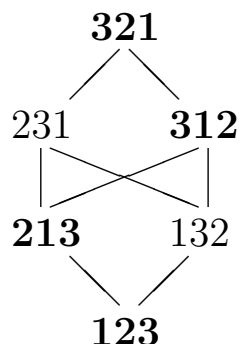

Figure 1: $\left(S_{3}, \leqslant\right)$

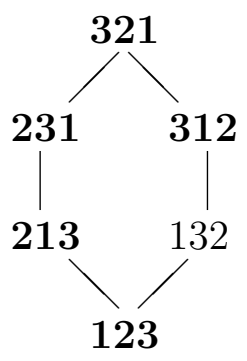

Figure 2: $\left(S_{3}, \leqslant w\right)$ 


\section{Invariant principal ideals under Han's map}

In this section, we show that in the Bruhat order, Han's bijection $H$ has the same invariant principal order ideals as Foata's transformation $\Phi$.

Theorem 7. Let $\sigma$ be a permutation in $S_{n}$. Then $\Lambda_{\sigma}$ is invariant under $H$ if and only if it is invariant under $\Phi$.

Proof. We first show that if $\Lambda_{\sigma}$ is invariant under $\Phi$ then it is invariant under $H$. We have the following two cases.

Case 1: $\sigma_{n}=n$. We use induction on $n$. Assume that the assertion is true for $S_{n-1}$. By Theorem 5, we see that $\Lambda_{\sigma^{\prime}}$ is invariant under $\Phi$, where $\sigma^{\prime}=\sigma_{1} \sigma_{2} \cdots \sigma_{n-1}$. Thus, by the induction hypothesis, we obtain that $\Lambda_{\sigma^{\prime}}$ is invariant under $H$. Notice that

$$
\Lambda_{\sigma}=\left\{\tau n: \tau \in \Lambda_{\sigma^{\prime}}\right\}
$$

Since $H(\pi n)=H(\pi) n$ for any permutation $\pi \in S_{n-1}$, we deduce that $\Lambda_{\sigma}$ is invariant under $H$.

Case 2: $\sigma_{n}=k<n$. Again, by Theorem 5, we see that

$$
\sigma=n(n-1) \cdots(k+1)(k-1) \cdots 21 k,
$$

which implies that $\Lambda_{\sigma}=\left\{\pi \in S_{n}: \pi_{n} \geqslant k\right\}$. Since the map $H$ preserves the last element of a permutation in $S_{n}$, we see that $\Lambda_{\sigma}$ is invariant under $H$.

Conversely, assume that $\Lambda_{\sigma}$ is invariant under $H$. We wish to show that $\Lambda_{\sigma}$ is invariant under $\Phi$. We also have the following two cases.

Case 1: $\sigma_{n}=n$. We proceed to use induction on $n$. Assume that the assertion is true for $S_{n-1}$. It is easy to see that

$$
\Lambda_{\sigma}=\left\{\tau n: \tau \in \Lambda_{\sigma^{\prime}}\right\}
$$

where $\sigma^{\prime}=\sigma_{1} \sigma_{2} \cdots \sigma_{n-1}$. So we have

$$
H\left(\Lambda_{\sigma}\right)=\left\{H(\tau n): \tau \in \Lambda_{\sigma^{\prime}}\right\}=\left\{H(\tau) n: \tau \in \Lambda_{\sigma^{\prime}}\right\}
$$

By the assumption that $\Lambda_{\sigma}$ is invariant under $H$, we see that $\Lambda_{\sigma^{\prime}}$ is invariant under $H$. Thus, by the induction hypothesis, we find that $\Lambda_{\sigma^{\prime}}$ is invariant under $\Phi$. Therefore,

$$
\begin{aligned}
\Phi\left(\Lambda_{\sigma}\right) & =\left\{\Phi(\tau n): \tau \in \Lambda_{\sigma^{\prime}}\right\} \\
& =\left\{\Phi(\tau) n: \tau \in \Lambda_{\sigma^{\prime}}\right\} \\
& =\left\{\tau n: \tau \in \Lambda_{\sigma^{\prime}}\right\} \\
& =\Lambda_{\sigma} .
\end{aligned}
$$

So we arrive at the assertion that $\Lambda_{\sigma}$ is invariant under $\Phi$.

Case 2: $\sigma_{n}=k<n$. We claim that

$$
\sigma=n(n-1) \cdots(k+1)(k-1) \cdots 1 k \text {. }
$$


To prove (10), we use induction on $k$. We first verify that (10) is valid for $k=n-1$. In this case, by an argument similar to the proof of Lemma 4, we may deduce that $\sigma$ is 132 -avoiding. This implies that $\sigma_{1}=n$. Assume that $\sigma_{n-1}=i$. It is easy to check that

$$
H(\sigma)_{n-1}=i+1, \quad H^{2}(\sigma)_{n-1}=i+2, \quad \ldots, \quad H^{n-i-1}(\sigma)_{n-1}=n, \quad H^{n-i}(\sigma)_{n-1}=1 .
$$

Since $\Lambda_{\sigma}$ is invariant under $H$, we see that $H^{n-i}(\sigma) \leqslant \sigma$, which implies $i=1$. Thus we have

$$
\sigma=n \sigma_{2} \cdots \sigma_{n-2} 1(n-1)
$$

To show that $\sigma$ is a permutation of form (10), we notice that the last element of the permutation $\tau=(n, n-1) \sigma$ is $n$. Clearly, $\Lambda_{\tau}=\left\{\pi: \pi \in \Lambda_{\sigma}, \pi_{n}=n\right\}$. So we deduce that $\Lambda_{\tau}$ is invariant under $H$. Let $\tau^{\prime}=\tau_{1} \cdots \tau_{n-1}$. Since $\tau_{n}=n$, by the assertion in Case 1 , we see that $\Lambda_{\tau^{\prime}}$ is invariant under $H$. Therefore, by the induction hypothesis, we conclude that $\Lambda_{\tau^{\prime}}$ is invariant under $\Phi$. In view of Theorem 5 , we have

$$
\tau^{\prime}=(n-1)(n-2) \cdots 21
$$

and hence

$$
\tau=\tau^{\prime} n=(n-1)(n-2) \cdots 21 n,
$$

which yields that $\sigma=n(n-2) \cdots 21(n-1)$. This completes the proof of $(10)$ in the case $k=n-1$.

We next consider the case $\pi_{n}=k<n-1$. Let

$$
\tau=(k, k+1) \sigma
$$

It is clear that $\tau=M(\sigma, k+1)$. By an argument analogous to the proof of relation (8), we deduce that

$$
\Lambda_{\tau}=\left\{\pi: \pi \leqslant \sigma, \pi_{n} \geqslant k+1\right\},
$$

from which it is easily seen that $\Lambda_{\tau}$ is invariant under $H$. Since $\tau_{n}>k$, by the induction hypothesis, we get

$$
\tau=n(n-1) \cdots(k+2) k(k-1) \cdots 21(k+1) .
$$

It follows that

$$
\sigma=(k, k+1) \tau=n(n-1) \cdots(k+2)(k+1)(k-1) \cdots 21 k .
$$

Thus the proof of (10) is complete.

By Theorem 5, we see that $\Lambda_{\sigma}$ is invariant under $\Phi$. This completes the proof.

\section{Acknowledgements}

We wish to thank Professor Guo-Niu Han for helpful suggestions. 


\section{References}

[1] A. Björner and F. Brenti, Combinatorics of Coxeter Groups, Graduate Texts in Mathematics, Vol. 231, Springer-Verlag, New York, 2005.

[2] A. Björner and M.L. Wachs, Permutation statistics and linear extensions of posets, J. Combin. Theory Ser. A, 58: 85-114, 1991.

[3] W.Y.C. Chen, N.J.Y. Fan and T.X.S. Li, Han's bijection via permutation codes, European J. Combin., 32: 217-225, 2011.

[4] C. Ehresmann, Sur la topologie de certains espaces homogénes, Ann. Math., 35: 396-443, 1934.

[5] D. Foata, On the Netto inversion number of a sequence, Proc. Amer. Math. Soc., 19: 236-240, 1968.

[6] G.-N. Han, Une courte démonstration d'un résultat sur la Z-statistique, C. R. Acad. Sci. Paris, Série I, 314: 969-971, 1992.

[7] D. Zeilberger and D.M. Bressoud, A proof of Andrews $q$-Dyson conjecture, Discrete Math., 54: 201-224, 1985. 